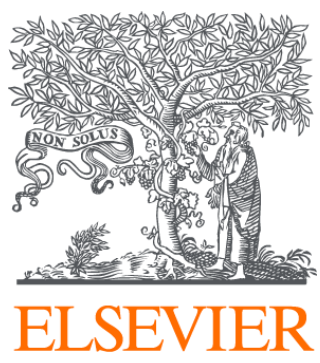

Since January 2020 Elsevier has created a COVID-19 resource centre with free information in English and Mandarin on the novel coronavirus COVID-

19. The COVID-19 resource centre is hosted on Elsevier Connect, the company's public news and information website.

Elsevier hereby grants permission to make all its COVID-19-related research that is available on the COVID-19 resource centre - including this research content - immediately available in PubMed Central and other publicly funded repositories, such as the WHO COVID database with rights for unrestricted research re-use and analyses in any form or by any means with acknowledgement of the original source. These permissions are granted for free by Elsevier for as long as the COVID-19 resource centre remains active. 


\section{Journal Pre-proofs}

Review

So much at stake: Ethical tradeoffs in accelerating SARSCoV-2 vaccine development

Christine Grady, Seema Shah, Franklin Miller, Marion Danis, Marie Nicolini, Jorge Ochoa, Holly Taylor, Dave Wendler, Annette Rid

PII: S0264-410X(20)31048-3

DOI: https://doi.org/10.1016/j.vaccine.2020.08.017

Reference: JVAC 22215

To appear in: $\quad$ Vaccine

Received Date: $\quad 19$ July 2020

Revised Date: $\quad 3$ August 2020

Accepted Date: $\quad 6$ August 2020

Please cite this article as: C. Grady, S. Shah, F. Miller, M. Danis, M. Nicolini, J. Ochoa, H. Taylor, D. Wendler, A. Rid, So much at stake: Ethical tradeoffs in accelerating SARSCoV-2 vaccine development, Vaccine (2020), doi: https://doi.org/10.1016/j.vaccine.2020.08.017

This is a PDF file of an article that has undergone enhancements after acceptance, such as the addition of a cover page and metadata, and formatting for readability, but it is not yet the definitive version of record. This version will undergo additional copyediting, typesetting and review before it is published in its final form, but we are providing this version to give early visibility of the article. Please note that, during the production process, errors may be discovered which could affect the content, and all legal disclaimers that apply to the journal pertain.

Published by Elsevier Ltd. 


\section{So Much at Stake: Ethical Tradeoffs in Accelerating SARSCoV-2 Vaccine Development}

Christine Grady a, Seema Shah ${ }^{b}$, Franklin Miller ${ }^{\mathrm{c}}$, Marion Danis ${ }^{\text {a }}$, Marie Nicolini a , Jorge Ochoa ${ }^{\mathrm{a}}$, Holly Taylor ${ }^{\mathrm{a}}$, Dave Wendler ${ }^{\mathrm{a}}$, Annette Rid ${ }^{\mathrm{a}}$

a Department of Bioethics, National Institutes of Health Clinical Center, Building 10/1C118, NIH, Bethesda MD 20892, cgrady@nih.gov; mdanis@ nih.gov; marie.nicolini@ nih.gov; jorge.ochoa@nih.gov; holly.taylor2@nih.gov; dwendler@cc.nih.gov; annette.rid@ nih.gov

b Lurie Children's Hospital, Northwestern University Department of Pediatrics, 225 E. Chicago Ave, Chicago, Illinois 60611, Seshah@lurie.childrens.org.

c Weill Cornell Medical College, Weill Cornell Medical College, 1300 York Ave, New York, NY 10065 fgm2002@med.cornell.edu

\section{Corresponding Author:}

Christine Grady, Department of Bioethics, NIH Clinical Center, Building 10/1C118, Bethesda MD 20892. 301-496-2429, cgrady@ nih.gov

Disclaimer: The views expressed are those of the authors and do not necessarily reflect those of the National Institutes of Health or the Department of Health and Human Services.

Funding. This work was partially funded by the National Institutes of Health $(\mathrm{NIH})$ Clinical Center, Department of Bioethics.

Key words: Vaccine, Ethics, clinical trials, SARS Co-V-2

Word count (text): 4220

Highlights

- Quickly finding a safe and effective vaccine against SARS Co-V-2 would be of great value

- It is ethically important to collect rigorous reliable data about vaccine safety and efficacy without compromising critical ethical and scientific norms.

- The best approach for obtaining rigorous data without compromising norms is accelerated individually randomized controlled trials.

- Widespread early distribution through an emergency use authorization may not serve public health goals nor the goal of identifying a safe, effective, reliable vaccine for SARS Co-V 2 


\title{
So Much at Stake: Ethical Tradeoffs in Accelerating SARSCoV-2 Vaccine Development
}

\begin{abstract}
Background: A sense of urgency exists to develop vaccines against SARS Co-V-2, responsible for numerous global cases and deaths, as well as widespread social and economic disruption. Multiple approaches have been proposed to speed up vaccine development, including accelerated randomized controlled trials (RCT), controlled human challenge trials $(\mathrm{CHI})$, and wide distribution through an emergency use authorization after collecting initial data. There is a need to examine how best to accelerate vaccine development in the setting of a pandemic, without compromising ethical and scientific norms.

Methods: Trade-offs in scientific and social value between generating reliable evidence about safety and efficacy while promoting rapid vaccine availability are examined along five ethically relevant dimensions: 1) confidence in and generalizability of data, 2) feasibility, 3) speed and cost, 4) participant risks, and 5) social risks.
\end{abstract}

Results: Accelerated individually randomized RCTs permit expeditious evaluation of vaccine candidates using established methods, expertise, and infrastructure. RCTs are more likely than other approaches to be feasible, increase speed and reduce cost, and generate reliable data about safety and efficacy without significantly increasing risks to participants or undermining societal trust.

Conclusion: Ethical analys is suggests that accelerated RCTs are the best approach to accelerating vaccine development in a pandemic, and more likely than other approaches to enhance social value without compromising ethics or science. RCTs can expeditiously collect rigorous data about vaccine safety and efficacy. Innovative and flexible designs and implementation strategies to respond to shifting incidence and test vaccine candidates in parallel or sequentially would add value, as will coordinated data sharing across vaccine trials. CHI studies may be an important complementary strategy when more is known. Widely disseminating a vaccine candidate without efficacy data will not serve the public health nor achieve the goal of identifying safe and effective SARS Co-V 2 vaccines. 
The novel severe acute respiratory syndrome coronavirus 2 (SARS-CoV-2) is raging globally with little diminution in sight, having infected more than 17 million people and killed close to 700,000 as of July 2020.[1] Intense public pressure and competition is fueling a race to find a safe and effective vaccine for SARS-CoV-2, as many see a vaccine as a necessary step in limiting infections and deaths, while allowing resumption of economic and other activities reminiscent of our pre-pandemic lives. While public health containment strategies and the passage of time could have some success in controlling the pandemic, some believe that "Without a coronavirus vaccine, we will never be able to live normally again. The only real exit strategy from this crisis is a vaccine that can be rolled out worldwide." [2] There are promises to accelerate vaccine development for SARS-CoV-2 at "pandemic speed" [3] or "warp speed" [4], driven by the idea that faster access to an effective vaccine could save or improve many lives [5]. We examine the ethics of commonly proposed approaches to accelerating vaccine development, focusing on trade-offs in scientific and social value between promoting rapid availability of a vaccine and generating reliable evidence about its safety and efficacy-while also attending to other important requirements of ethical research.

\section{Vaccine development during the SARS-CoV-2 pandemic}

Scientists leapt into SARS-CoV-2 vaccine research within weeks of identifying and sequencing the novel coronavirus [6]. Several factors gave scientists a head start: 1) an "... explosion in basic scientific understanding, including in areas of genomics and structural biology, ...supporting a new era in vaccine development" [3]; 2) established vaccine platforms and newly developed ones, for example RNA and DNA vaccines and recombinant sub-unit vaccines; 3) vaccine research against two other coronaviruses - the severe acute respiratory syndrome (SARS) and middle east respiratory syndrome (MERS) viruses - that, however, did not advance beyond phase I studies; [7] and 4) vaccine research experiences during recent epidemics, notably Ebola in 2014-16 and Zika in 2015-16 [8]. By the end of July 2020, more than 160 SARS- CoV-2 vaccine candidates were in the pipeline, several already entered phase 1 or 1-2 clinical trials [9], and a few candidates began phase 3 trials in summer 2020. [10]

Governments, the World Health Organization, and major non-governmental organizations such as the Coalition for Epidemic Preparedness Innovation (CEPI), the Wellcome Trust, and the Gates Foundation, have encouraged vaccine scientists to explore various approaches for 
accelerating vaccine development $[3,5,11]$. Options being discussed include moving directly to randomized controlled efficacy trials (RCT) after phase 1 [12] with either traditional individually randomized or cluster randomized designs [13]; controlled human infection (CHI) or "human challenge" studies [14]; innovative adaptive designs that allow parallel testing of multiple candidates [15]; and wide distribution of a vaccine through mechanisms like an Emergency Use Authorization (EUA) ${ }^{1}$ after phase 1 or II clinical data, among others $[12,16]$. Efforts are bolstering vaccine trial acceleration while research on vaccine candidates is in progress, such as developing novel cell-line models, forgoing animal studies, establishing cohorts of potential participants and possible testing sites, and establishing public-private partnerships [7, 17]. Pharmaceutical companies are building manufacturing capacity for rapid production of candidates with the goal of producing billions of vaccine doses by 2021 in advance of the conduct of pivotal efficacy trials. These companies have received generous support from the U.S. government, and are also investing their own funds at great financial risk [12].

Widespread fear, disruptions and illness caused by the SARS-CoV-2 pandemic and the hope and perceived need for a vaccine create pressure to skip steps or accept certain compromises in the search for a vaccine. Although shortcuts in vaccine development and testing might expedite the timeline of scientific progress, they could also result in compromising quality, acceptability, and ethics. As others have noted, “...what cannot and must not be allowed is for desperation to result in the suspension of scientific principles and ethical research values" [18]. In what follows, we apply ethical considerations to vaccine research in the context of a pandemic, focusing primarily on social value and scientific validity. We contrast proposed approaches to accelerate SARSCoV-2 vaccine development, identifying major ethical trade-offs involved.

\section{Ethics and Vaccine Development}

In normal circumstances, vaccine clinical trials present distinct challenges compared to treatment trials. Classic clinical efficacy trials of vaccines typically enroll thousands or tens of thousands

\footnotetext{
${ }^{1}$ Multiple recent reports from around the globe describe plans to dis seminate vaccines before efficacy trials are complete, e.g. https://www.technologyreview.com/2020/07/29/1005720/george-church-diy-coronavirus-vaccine/); (www.wsj.com > articles > russia-seeks-to-register-first-cor...); https://www.nytimes.com/2020/07/16/bus iness/chinavaccine-coronavirus.html; https://www.scmp.com/week-asia/health-environment/article/3095371/indias-rushcoronavirus-vaccine-being-driven-national). The FDA says it will decide on a case by case basis .
} 
of otherwise healthy uninfected volunteers who are not sick and not in danger of immediate illness or death. These healthy participants accept some vaccine risk primarily for the public good. Personal benefit to study participants is uncertain, not only because protective efficacy is still unknown in research and some participants will receive placebo, but also because not every vaccinated person would be exposed or infected even without a vaccine. The primary ethical concern is to find a safe and effective vaccine for persons at risk of infection, while not exposing study participants to unnecessary risk in the process [19].

Investigating vaccines is typically a slow, lengthy and stepwise process that prioritizes safety. The process begins with proof of concept studies that try to mimic natural protection, then animal studies, small dose finding studies, evaluation of safety and immunogenicity, and ultimately prospective large-scale randomized controlled clinical trials to test efficacy and then attain licensure. Although it is difficult to calculate precisely, the average time to develop vaccines is estimated to be 10 years or more [20]. Moreover, many vaccine concepts never progress into clinical testing at all; and at best, only about $15-30 \%$ of vaccine candidates that enter phase 1 are ultimately licensed [21]. Vaccines for certain microbes including HIV and malaria have remained elusive, despite decades of research [22]. Other vaccines, such as existing seasonal influenza vaccines, afford a limited duration of protection only against specific strains.

In addition to minimizing risk for healthy research volunteers and the general public, a cautious approach to vaccine development is also a response to past missteps and adverse events emerging late in the development process. Pressure to develop vaccines due to a sense of public health urgency has occasionally backfired. A hastily tested vaccine in the 1930 s resulted in several deaths and numerous cases of vaccine-associated paralytic polio, halting polio vaccine research for more than 15 years. Later, even after the large and successful (albeit controversial) randomized trial of Salk's inactivated polio vaccine in children and the vaccine's quick licensure, a contaminated vaccine lot led to unexpected events and deaths, and the polio vaccine program was paused yet again [23]. In 1976, the US government quickly produced and widely distributed a swine flu vaccine in response to fear of a new influenza pandemic, following pandemics in 1957 and 1968. That influenza pandemic never materialized, children experienced a high rate of adverse reactions to the vaccine, and more than 500 vaccinated persons developed 
vaccine-induced Guillian Barre syndrome [24]. More recently, two large randomized trials of an adenovirus 5 (Ad 5) vector HIV vaccine were terminated early after finding that vaccinees who had high preexisting antibody titers against Ad5 were more likely to become HIV infected [25]. Missteps in handling emerging data from a novel dengue vaccine about significant diseaseenhancement risks in those without previous dengue exposure resulted in confusion, anger, distrust, and decreased uptake of all vaccines in the Philippines. [26]

Moreover, vaccines have always been controversial, despite the history of being quite safe and remarkably successful at significantly abating some formidable human diseases, such as smallpox, polio, yellow fever, and measles [27]. Public concerns about vaccines include concerns about product safety and untoward effects, disturbing the natural order, mandating individual vaccination for the public good, inequity in access to the health benefits of vaccines, and others. Vaccines must be extremely safe and effective for public acceptability and population health. Even then, fear of vaccines, public mistrust, vaccine hesitancy, and anti-vaccine activism remain. [28]

\section{Ethics and Vaccine Development during a pandemic}

Vaccine development during recent epidemics such as SARS, H1N1, Ebola, and Zika imparted lessons about the ethics of conducting research during pandemics, including the importance of rigorous research, the complexities of targeting high risk participants, the necessity of extensive social engagement and mobilization, and the implementation of innovative designs, especially as patterns of infection shifted. Despite debates about ethics, feasibility, and social acceptability of conducting vaccine research during a public health emergency, many concluded that research is an essential component of an epidemic response and should be timely, scientifically reliable, feasible, and ethically responsible [29]. The sense of urgency and desire to stave off the SARSCoV-2 pandemic by accelerating vaccine development raise similar issues about research during a public health emergency and the tension between speed and rigor.

Among widely accepted requirements of ethical research [30], two requirements- social value and scientific validity- warrant primary consideration in determining the ethical acceptability of approaches to accelerating vaccine development. The urgency of the current pandemic could 
lead to compromising either or both of these requirements [31]. In what follows, we examine the social and scientific value, risks, and costs of each proposed approach. To focus our attention on social and scientific value, risks and costs, we set aside discussion of other important ethical requirements for human research that also should be addressed, including informed consent, fair participant selection, minimizing risks, enhancing respect for enrolled participants, community engagement, access to experimental interventions, and scale up and planning for global fair access of vaccines shown to be effective [30].

Ethical analysis involves evaluating tradeoffs between speed and rigor for each proposed vaccine development acceleration approach and the resultant impact on social and scientific value. Ethically acceptable tradeoffs should be iteratively re-evaluated amidst rapidly changing pandemic conditions, for example changes in incidence or epidemiological patterns, the emergence of treatments, and the benefits of public health measures. In general, ethically acceptable tradeoffs are those that permit the generation of rigorous reliable data about safety and efficacy in an accelerated way without compromising critical ethical and scientific norms, as detailed below.

\section{Scientific and social value of accelerating vaccine development}

A safe and effective vaccine for SARS-CoV-2 could have great social value in reducing disease, given the prevalence and transmissibility of SARS-CoV-2, the range of morbidity and mortality associated with COVID-19, and the health, economic, and social costs of the global pandemic. The anticipated social value of a SARS-CoV-2 vaccine support responsibly accelerating vaccine development. Speed adds value because the sooner a safe and effective vaccine is identified, the sooner people could be protected from COVID-19, and many lives could be improved or saved. Exactly how many lives would depend on how protective the vaccine is, how many people actually get it, patterns of infection and disease, and other factors. Importantly, the perceived need for urgency could change. The sense of urgency could wane if one or more safe, effective, and accessible treatments are identified; if public health strategies, such as physical distancing and mask wearing, are adhered to, effective, and sustainable; and/or if the incidence of COVID19 significantly drops. On the other hand, the sense of urgency could stay the same or increase if 
one or more of these does not occur and serious illness, economic devastation, and social disruption persists.

It is possible, perhaps likely, that no single vaccine among the multiple candidates in the pipeline will prove to be safe and effective across all at risk groups or settings. Multiple candidates with different product profiles and manufacturing requirements increase the probability of a successful, affordable, and valuable vaccination strategy, as long as there is good evidence of safety and efficacy across sample populations. The social value of having one or more SARSCoV-2 vaccines will partly depend on how safe and effective the vaccine candidates are, and the degree of public confidence. An overall mitigation strategy for COVID-19 might combine one or more, even partially effective, vaccines with public health strategies and other prevention modalities like monoclonal antibodies or treatment as prevention [32].

\section{Scientific and social risks and costs of accelerating vaccine development}

Accelerating vaccine development also could entail ethically unacceptable shortcuts and tradeoffs. Rushing forward without sufficient rigor and coordination could lead to uncertainty and lack of confidence about the risks and benefits of particular vaccine candidates and undermine the success of any vaccination effort. Social value, acceptability, and public confidence in a vaccine could be jeopardized if rushing results in partial, inadequate, or unreliable data about vaccine safety or efficacy, deployment of SARS Co-V 2 vaccines that are insufficiently protective, protective in only certain groups or for only a short time, or result in enhancement of infection or disease [33]. Unexpected findings, especially harm from unforeseen or unacceptable side effects or adverse events that might emerge late in vaccine development (or after widespread vaccine distribution), could also jeopardize social value and acceptability and amplify overall public distrust in vaccines. Any of these shortcomings could intensify already pervasive public anti-vaccine sentiment [34].

Furthermore, competition and rapid testing of vaccine candidates without sufficient attention to rigor, ethics, and coordination could waste vital and limited vaccine research resources, including funding, trained research staff, and study participants. Premature or misleading information including reporting findings before they are adequately vetted could jeopardize trust 
[35]. Premature distribution of a partially safe or effective vaccine candidate could give people a mistaken view of how protected they are and encourage risky behaviors, or distract from public health measures. Premature distribution could also muddy the waters for future testing, as an early vaccine candidate might become the standard of prevention and thus delay or hamper finding a safer or more effective vaccine.

\section{Comparative evaluation of tradeoffs in social and scientific value and costs}

Evaluation of different approaches to accelerating vaccine development involves assessing tradeoffs in scientific and social value along five ethically relevant dimensions: 1) confidence in and generalizability of the data, 2) feasibility, 3) speed and cost, 4) risks to participants, and 5) social risks, including opportunity costs. We consider these dimensions below and in the Table, while recognizing that even within each approach, the details of vaccine trials will vary, influencing design features, outcomes, and ultimately social value.

[Insert Table]

\section{Confidence in and generalizability of the data}

Confidence in the data and thus its social value will depend on the rigor and generalizability of the data and may differ between approaches. Generalizability of safety and efficacy data to those most likely to benefit from vaccination depend on who is enrolled in the trials. Including trial participants of multiple ages, susceptibilities, and geographies enhances the generalizability of findings to various populations and risk levels. Phase 1 trials usually enroll a small number of healthy persons who are at low risk of infection, while standard phase 3 vaccine efficacy trials enroll a large cohort of healthy persons at higher risk of infection. Accelerated individually randomized controlled trials and cluster randomized trials would similarly expect to enroll healthy persons at high risk and could target specific high risk groups to enhance generalizability. RCTs are likely to include participants from known risk groups such as those who are older, have chronic illnesses, and are at high occupational risk (e.g. healthcare workers, meat-packing plant employees) who are expected to use and benefit from an eventual vaccine. Two vaccine approach options would provide less generalizability: 1) CHI studies of vaccines through deliberate infection of a small number of young, healthy people, and 2) moving directly to wide distribution of vaccine to people at all risk levels (not in research) through an EUA after 
only phase 1 or 2 data. Since serious complications and mortality from COVID-19 appear to be lower in young people without comorbidities, vaccine data from populations typically enrolled in phase I trials or CHI studies may not be generalizable to those most in need of protection from a SARS-CoV-2 vaccine $[36,16]$.

\section{Feasibility}

Feasibility is another important consideration for scientific validity and social value in vaccine development. Feasibility of conducting vaccine trials typically depends on the incidence of infection, as declining incidence can make demonstrating vaccine efficacy difficult. Accelerated individually randomized or cluster-randomized RCTs, similar to standard vaccine development approaches, rely on incidence to determine efficacy. Since this accelerated RCT approach aims to get safety and efficacy data fast, however, it may have a better chance of obtaining needed data before any significant decrease in incidence occurs naturally or as the result of effective public health measures. Recent plans to include Brazil in SARS-CoV-2 vaccine RCTs from the United Kingdom and from China are examples of responding to changes in incidence, as cases are rapidly increasing in Brazil in June 2020 [37]. Similarly, some trial design strategies allow enrolling high-risk participants even in the setting of declining incidence. The 2015 Ebola ring vaccination trial in Guinea, for example, was successful in recruiting participants at high-risk during a waning epidemic by recruiting an epidemiologically defined ring of contacts (and contacts of contacts) of newly diagnosed Ebola patients [38]. CHI studies are even less dependent on population incidence, as data in these studies are collected from participants infected in a controlled way. Incidence also would not necessarily influence vaccine distribution through an EUA, as there is not an intention to evaluate efficacy, and vaccine would be available to people even if SARS Co-V 2 was to significantly diminish.

\section{Speed and cost}

With regard to speed and cost, carefully executed RCTs, even accelerated, require many months, thousands of participants, and significant financial investment to gather sufficient and reliable data. The necessary expertise and infrastructure for an accelerated individually randomized trial is already in place, whereas cluster randomized designs often require additional specialized expertise, especially statistical, and potentially novel infrastructure. CHI studies offer what some 
believe is a faster approach to finding a vaccine than accelerated RCTs [39]. CHI studies, however, initially require the development of a reliable SARS-CoV-2 CHI model using good manufacturing practices as well as testing for validity and appropriate dosing, scientific expertise, and the preparation of unique organized sites and infrastructure [40]. Depending on how long it takes to set up the CHI model and how quickly accelerated RCTs can progress, RCTs might be faster than the initial time required to begin a $\mathrm{CHI}$ study [41]. Further, vaccines identified as promising in CHI studies generally require later field testing in RCTs. However, once a CHI model and infrastructure are in place, and more is known about the disease, $\mathrm{CHI}$ could be useful to test promising vaccine candidates in a small number of persons to help prioritize vaccine candidates before proceeding to larger efficacy trials. Distributing one or more vaccine candidates, under EUA for example, after collecting preliminary data is the fastest way to get vaccine candidates to a large segment of the general public. Distributing a vaccine candidate in this way is not research, but would be done with public health goals in mind. Whether or not such rapid distribution would meet public health goals of mitigating SARS-CoV2 will depend on how safe the vaccine candidate is, how well it protects against COVID-19, and how it might change behaviors, all of which would be relatively unknown and difficult to measure once a vaccine is widely available. Vaccine manufacturers are making efforts and promises to produce billions of doses of vaccines before efficacy testing, so that vaccines will be ready for wide distribution. Although the US and others are providing funds for this, scaling up now presents a major financial risk as some vaccine candidates are likely to fail to be efficacious or safe.

\section{Risks to participants and participant numbers}

Considering risks to participants and vaccinated individuals is also critical in ethically evaluating each potential approach to vaccine testing. Accelerating an individual or cluster randomized trial includes a possible increased chance of unforeseen adverse events. In an RCT, risks to individual participants are carefully monitored and minimized and common side effects are likely to be well characterized. Nonetheless, due to a shorter timeframe and fewer participants, rarer and possibly serious side effects may be less likely to be seen. Rare side effects are even less likely to be identified in CHI studies or in wide EUA distribution because of the small number of evaluated participants. $\mathrm{CHI}$ studies pose more deliberate risk to individual participants 
than other approaches; despite emerging data, there remain unanswered questions about short and long term consequences of infection or about any effective way to mitigate or reverse symptoms in infected persons. At the same time, fewer people overall might be exposed to risks from the vaccines themselves in CHI studies than those given vaccine in RCTs. Widespread distribution of vaccine candidates via EUA based on preliminary data has the potential to expose large numbers of people to unknown and possibly serious risks not observed in early trials, including the possibility of antibody dependent disease enhancement. Even with mandated safety monitoring after EUA distribution, it would be difficult or impossible to ascertain vaccineinduced adverse events, the extent to which host factors like age, underlying illness, or use of medications intensified the chance of adverse events, how safe the vaccine candidate was across different groups, or the effects of possibly increased risky behaviors after vaccination. Many people might be harmed before a widely distributed vaccine candidate could be pulled.

\section{Social risks, including opportunity costs}

Social risks, including public perception and trust in vaccines will depend not only on confidence in the safety of the vaccine and its ability to protect people from COVID, but also on confidence in how data were generated, and public engagement in and awareness of the vaccine trial process. The selection of outcome measures, perceived fairness in selection of participants or sites, any harm to trial participants, the process of monitoring and following participants, and other features could all affect how much people trust the results. Because deliberate infection is counterintuitive and generally hard for the public to understand, a serious adverse event or death in a CHI study could threaten public trust in vaccine research more broadly. Public engagement to enhance awareness about how vaccines are developed and what to realistically expect from each approach could bolster public confidence in resultant vaccines. Community engagement, social mobilization, and dialogue-based interventions are critical for bolstering public acceptance and trust in vaccines, and ultimately for vaccine utilization [42]. This is especially important when there is widespread fear and uncertainty and limited public trust in vaccines.

Accelerating the first vaccine candidates determined to be safe into RCTs could result in opportunity costs and delay the testing of more promising- safer or more effective- candidates [43]. A carefully coordinated and flexible study design, perhaps using a platform or other 
approach to test vaccine candidates in parallel or sequentially, might help to mitigate these downsides. Similarly careful coordination and sharing of data across studies, perhaps with a well constituted Data Monitoring Committee (DMC) could facilitate decisions about stopping vaccine candidates that have unacceptable side effects or accelerating candidates that show the most promise in protecting against COVID-19. Later, carefully planned and conducted CHI studies might facilitate rapid testing of future vaccine candidates in combination with accelerated efficacy trials, but would require considerable public engagement.

Confidence would likely be low in the safety and efficacy of a vaccine candidate that was widely distributed under a EUA after only early phase testing that assessed preliminary safety and immunogenicity, but not efficacy. The public might wrongly believe that they are protected by receiving a widely distributed vaccine when, in fact, it will not be known how protective it is and persons might relax other protective public health methods exposing themselves to greater risk of harm. It would be unclear whether or how to attribute epidemiologic changes to distributed vaccine candidates without any comparator, especially as the pandemic fluctuate $s$ in different locations without any vaccine. Wide distribution under a EUA also presents important opportunity costs, increasing the difficulties of testing vaccine candidates which might be safer or more protective. Moreover, a vaccine distributed under a EUA has the potential to become the unproven standard for prevention, creating pressure for other trials to use it as the comparator and making it difficult to interpret subsequent trial results. For these reasons, a EUA strategy for vaccine distribution without prior efficacy evaluation is not ethically acceptable.

\section{Conclusion}

Our analysis of the likely social and scientific value of each approach in relation to its social and scientific risks and costs suggests that accelerated individually randomized RCTs are most likely to enhance social value without compromising ethics or science. RCTs allow collection of rigorous data about vaccine candidate safety and efficacy while expediting the process, minimizing harm, and controlling costs. Such RCTs should consider innovative and flexible designs and implementation strategies that can weather changing incidence of infection, test vaccine candidates in parallel or sequentially as they become available, using similar endpoints, stringent monitoring plans, and coordinated sharing of data collected from all vaccine trials to 
expedite identification of safe and effective vaccines. In the future, when more is known about SARS-CoV-2 susceptibility, pathophysiology, and clinical management, and the essential infrastructure is in place, $\mathrm{CHI}$ studies for vaccines may be an important complementary strategy to RCTs. A coordinated research effort could combine approaches with the goals of expeditiously finding one or more safe and effective vaccine candidates in a rigorous, transparent, and safe manner before wide deployment. Widely disseminating a vaccine candidate after only preliminary safety and immunogenicity data is ethically unacceptable and may not serve the public health nor achieve the goal of identifying a safe, effective, reliable vaccine for SARS Co-V 2.

Given the ravaging global effects of SARS Co-V-2, it is reasonable and valuable to accelerate testing vaccines for SARS-CoV-2. At the same time, rigorous data collection and analysis are critical to engender confidence in the safety and efficacy of any vaccine and consequent public trust in deployed vaccines. Multiple options for accelerating the testing and identification of a safe and effective vaccine should be considered and weighed on their scientific and ethical merits. Tradition, inflexibility, turf wars, and any reluctance to share data should be relinquished in favor of working towards a common goal without compromising safety, ethics, or confidence in research results. Ethically, it is essential to maximize the social and scientific value of such research and minimize the social and scientific costs and risks. 
[1] Johns Hopkins University, Coronavirus Resource Center. Available at https://coronavirus.jhu.edu/map.html Accessed August 2, 2020.

[2]. Draulans D. Finally, a virus got me.' Scientist who fought Ebola and HIV reflects on facing death from COVID-19. Science Magazine, May 8, 2020

[3] Lurie N, Saville M, Hatchett R, Haton J. Developing Covid-19 Vaccines at Pandemic Speed. N Engl J Med 2020; 382:1969-1973

[4] Cohen J. U.S. "Warp Speed" vaccine effort comes out of the shadows. Science. 2020; 368(6492): 692-693; AND Department of Health and Human Services, HHS Fact Sheet: Operation Warp Speed. June 16, 2020. https://www.hhs.gov/about/news/2020/06/16/fact-sheetexplaining-operation-warp-speed.html

[5] Gates B. What you need to know about the COVID-19 vaccine. GatesNotes, April 30, 2020. Available at https://www.gatesnotes.com/Health/What-you-need-to-know-about-the-COVID-19vaccine.

[6] Zhu N, Zhang D, Wang W, Li X, Yang B, Song J, Zhao X, Huang B, Shi W, Lu R, Niu P, Zhan F, Ma X, Wang D, Xu W, Wu G, Gao G, Tan W, for the China Novel Coronavirus Investigating and Research. A Novel Coronavirus from Patients with Pneumonia in China, $N$ Engl J Med 2020;382:727-33. DOI: 10.1056/NEJMoa20010172019 Team https:/www.nejm.org/doi/pdf/10.1056\%2FNEJMoa2001017

[7] Diamond M, Pierson T. The Challenges of Vaccine Development against a New Virus during a Pandemic. Cell Host and Microbe. 2020; 27(5): 699-703.

https://doi.org/10.1016/j.chom.2020.04.021

[8] Kieny MP, Salama P. WHO R\&D Blueprint: A Global Coordination Mechanism for R\&D Preparedness. Lancet. 2017; 389(10088):2469-2470. doi:10.1016/S0140-6736(17)31635-5. AND Platform for European Preparedness against (Re)emerging Epidemics https:/www.prepareeurope.eul

[9] Bioworld, Biopharma products in development for COVID-19 Available at https:/www.bioworld.com/COVID19products\#vac; AND Thanh Le T, Andreadakis Z, Kumar A, Gómez Román R, Tollefsen S, Saville M, Mayhew S. The COVID-19 vaccine development landscape Nature Reviews: Drug Discovery. 2020; 19: 305-306

[10] Cohen J. Pandemic vaccines are about to face the real test. Science 19 Jun 2020:

Vol. 368, Issue 6497, pp. 1295-1296. DOI: 10.1126/science.368.6497.1295

https://science.sciencemag.org/content/368/6497/1295

[11] World Health Organization. Access to COVID-19 Tools (ACT) Accelerator, April 24, 2020. https://www.who.int/who-documents-detail/access-to-covid-19-tools-(act)-accelerator AND Cohen J. With record-setting speed, vaccine makers take their first shots at the new coronavirus. Science. March 31, 2020. doi:10.1126/science.abb9996 [12] U.S. Department of Health and Human Services. HHS Accelerates Clinical Trials, Prepares for Manufacturing of COVID-19 Vaccines, March 30, 2020. Available at https:/www.hhs.gov/about/news/2020/03/30/hhs-accelerates-clinical-trials-preparesmanufacturing-covid-19-vaccines.html. Accessed July 10, 2020. AND U.S. Department of Health and Human Services Food and Drug Administration Center for Biologics Evaluation and Research. Development and Licensure of Vaccines to Prevent COVID-19: Guidance for Industry, June 2020; available at https:/www.fda.gov/media/139638/download 
[13] Kahn R, Rid A, Smith PG, Eyal N, Lipsitch M. Choices in vaccine trial design in epidemics of emerging infections. PLoS Med. 2018;15(8): e1002632.

https://doi.org/10.1371/journal.pmed.1002632

[14] Shah S, Miller F, Darton T, Duenas D, Emerson C, Lynch HF, Jamrozik E, Jecker N, Kamuya D, Kapulu M, Kimmelman J, MacKay D, Memoli M, Murphy S, Palacios R, Richie T, Roestenberg M, Saxena A, Saylor K, Selgelid M, Vaswani V, Rid A. Ethics of controlled human infection to address COVID-19. Science. 2020: 368 (6493): 832-834.

DOI: $10.1126 /$ science.abc1076

[15] World Health Organization. An international randomised trial of candidate vaccines against COVID-19.28 May 2020. Available at https $/ / w w w . w h o . i n t / p u b l i c a t i o n s-d e t a i l / a n-$ international-randomised-trial-of-candidate-vaccines-against-covid-19. Accessed July 12, 2020. AND Angus, D.C., Alexander, B.M., Berry, S. et al. Adaptive platform trials: definition, design, conduct and reporting considerations. Nat Rev Drug Discov 2019;18: 797-807. https://doi.org/10.1038/s41573-019-0034-3 https://www.nature.com/articles/s41573-019-0034-3 [16] Karlin-Smith S. COVID-19 Vaccines: Ex-FDA Leaders Urge High Bar for Emergency Use Authorization. Available at https://pharmaintelligence.informa.com/resources/productcontent/covid-19-vaccines-ex-fda- leaders-urge-high-bar-for-emergency-use-authorization; AND https://www.jnj.com/johnson-johnson-announces-a-lead-vaccine-candidate-for-covid-19landmark-new-partnership-with-u-s-department-of-health-human-services-and-commitment-tosupply-one-billion-vaccines-world wide-for-emergency-pandemic-use [17] Corey L, Mascola JR, Fauci AS, Collins FS. A Strategic Approach to COVID-19 Vaccine R \& D. Science. 2020; 368 (6494): 948-950. DOI: 10.1126/science.abc5312

AND NIH, Accelerating COVID-19 Therapeutic Interventions and Vaccines (ACTIV). Available at https://www.nih.gov/research-training/medical-research-initiatives/activ. Accessed June 30, 2020.

[18] Trogen B, Oshinsky D, Caplan A. Adverse consequences of rushing a SARS Co-V2 Vaccine. JAMA on line May 26, 2020

[19] Grady, C. Ethics of Vaccine Research. Nature Immunology 2004; 5(5):465-468

[20] Pronker ES, Weenen TC, Commandeur H, Claassen EHJHM, Osterhaus ADME (2013)

Risk in Vaccine Research and Development Quantified. PLoS ONE, 2013. 8(3): e57755. https://doi.org/10.1371/journal.pone.0057755. AND Heaton P. The COVID-19 Vaccine

Multiverse. New Eng J Med. July 14, 2020. DOI: 10.1056/NEJMe2025111

[21] Wong, Chi Heem, Kien Wei Siah, and Andrew W. Lo. "Estimation of clinical trial success rates and related parameters." Biostatistics 20.2 (2019): 273-286.; AND DiMasi, J. A., Florez, M. I., Stergiopoulos, S., Peña, Y., Smith, Z., Wilkinson, M., \& Getz, K. A. (2020). Development times and approval success rates for drugs to treat infectious diseases. Clinical Pharmacology \& Therapeutics, 107(2), 324-332.

[22] Plotkin SA. Vaccines we need but don't have. Viral Immunology. 2018; 31(2): 114-116. DOI: $10.1089 /$ vim.2017.0122018

[23] Plotkin SL, Plotkin SA. A short history of vaccination. Chapter 1 in Stanley Plotkin, Walter Orenstein, Paul Offit, Kathryn Edwards (eds). Plotkin's Vaccines. NY: Elsevier, 2018. Pages 115.

[24] Neustadt R, Fineberg H. The Epidemic That Never Was: Policy-Making and the Swine Flu Scare. NY: Vintage Books, 1983.

[25] Gray G, Buchbinder S, Duerr A. Overview of STEP and Phambili trial results: two phase IIb test of concept studies investigating the efficacy of MRK ad5 gag/ pol/nef sub-type B HIV 
vaccine. Curr Opin HIV AIDS. 2010; 5(5): 357-361. doi:10.1097/COH.0b013 https:/www.ncbi.nlm.nih.gov/pmc/articles/PMC2995949/pdf/nihms-229595.pdf [26] Plotkin SA Dengue Vaccine, a Double-Edged Sword. J Pediatric Infect Dis Soc. $2020 \mathrm{Apr}$ 30;9(2):107-109. doi: 10.1093/jpids/piy140.

[27] Destefano F, Offit P, Fisher A. Vaccine Safety. Chapter 82 in Stanley Plotkin, Walter Orenstein, Paul Offit, Kathryn Edwards (eds). Plotkin's Vaccines. NY: Elsevier, 2018. Pages: 1584-1600.

[28] World Health Organization. Report of the SAGE Working Group on Vaccine Hesitancy. 2014. Available at https://www.who.int/immunization/sage/meetings/2014/october/1_Report_WORKING_GROUP _vaccine_hesitancy_final.pdf. AND, Larson HJ, Jarrett C, Eckersberger E , Smith D, Paterson P. Understanding vaccine hesitancy around vaccines and vaccination from a global perspective: a systematic review of published literature, 2007-2012. Vaccine 2014 Apr 17;32(19):2150-9. : https://doi.10.1016/j.vaccine.2014.01.081

[29] Lipsitch M, Eyal N. Improving vaccine trials in infectious disease emergencies. Science.2017; (6347), 153-156.357. DOI: https//doi.10.1126/science.aam833; AND Edwards K, Kochhar S. Ethics of Conducting Clinical Research in an Outbreak Setting. Annu.Rev.Virol. 2020; 7:3.1-3.20 https://doi.org/10.1146/annurev-virology-013120013123; AND Natl. Acad. Sci. Eng. Med. 2017. Integrating Clinical Research into Epidemic Response: The Ebola Experience. Washington, DC: Natl. Acad. Press. Available at https:/www.nap.edu/catalog/24739/integrating-clinical-research-into-epidemic-response-theebola-experience. AND Nuffield Council on Bioethics. Research in Global Health Emergencies. January 2020. Available at https $/ /$ www.nuffieldbioethics.org/publications/research-in-globalhealth-emergencies/read-the-short-report/emergency-preparedness-response-and-research. AND Kahn R, Rid A, Smith PG, Eyal N, Lipsitch M (2018) Choices in vaccine trial design in epidemics of emerging infections. PLoS Med 15(8): e1002632.https//doi.org/10.1371/journal. pmed. 1002632

[30] Emanuel E, Wendler D, Grady C. An Ethical Framework for Biomedical Research. Chapter 11 in E. Emanuel, C. Grady, R. Crouch, R. Lie, F. Miller, D. Wendler (eds). The Oxford Textbook of Clinical Research Ethics. New York: Oxford U. Press. 2008 [31] London AJ, Kimmelman J. Against pandemic research exceptionalism. Science. 2020; 368(6490): 476-477. DOI: 10.1126/science.abc1731.

[32] Cohen M, Corey L. Combination prevention for COVID-19, Science. 2020; 368 (6491): 551. DOI: $10.1126 /$ science.abc5798.

[33] Graham B. Rapid COVID-19 vaccine development. Science. 2020; 368(6494):945-946. doi: $10.1126 /$ science.abb8923

[34] Walsh B. The viral spread of anti-vaccination sentiment, May 13 2020. Available at https:/www.axios.com/vaccine-coronavirus-pandemic-protest-b0ae67a4-1a12-4904-aec19cbc612ff8a0.html. AND, Jamison, P. Anti-vaccination leaders fuel black mistrust of medical establishment as covid-19 kills people of color. Washington Post July 17, 2020. Available at: https://www.washingtonpost.com/dc-md-va/2020/07/17/black-anti-vaccine-coronavirustuskegee-syphilis/. Accessed July 17, 2020

[35] CNBC Why this former Harvard Med prof says Moderna's vaccine trial is 'publication by press release'. May 20, 2020. Available at https://www.youtube.com/watch? v=Nz_D_t6kcPs. Accessed July 10, 2020. 
[36] Verity R, Okell L, Dorigatti I, Winskill P, Whittaker C, Imai N, et al. Estimates of the severity of coronavirus disease 2019: a model-based analysis. Lancet Infectious Disease. 2020; 20 (6):669-677. https://www.thelancet.com/journals/laninf/article/PIIS1473-3099(20)302437/fulltext

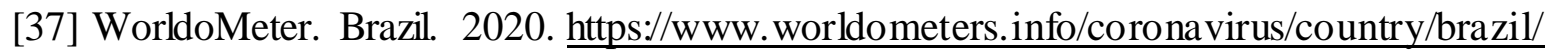
Accessed August 2, 2020

[38] Rid A, Miller FG. Ethical Rationale for the Ebola "Ring Vaccination" Trial Design Am J Public Health. 2016; 106(3): 432-435. doi: 10.2105/AJPH.2015.302996

[39] Eyal N, Lipsitch M, Smith PG, Human Challenge Studies to Accelerate Coronavirus Vaccine Licensure. J Infect Dis. 2020; 221(11):1752-1756. doi: 10.1093/infd is/jiaa152. [40] Darton TC, Blohmke C, Moorthy BS, Altmann DM, Hayden FG, Clutterbuck E, Levine M, Hill A, Pollard A. Design, recruitment, and microbiological considerations in human challenge studies. Lancet Infect Dis. 2015; 15(7):840-51. doi: 10.1016/S1473-3099(15)00068-7. AND Catchpole AP, Fullen DJ, Noulin N. et al. The manufacturing of human viral challenge agents for use in clinical studies to accelerate the drug development process. BMC Res Notes 11, 620 (2018). https://doi.org/10.1186/s13104-018-3636-7

[41] Deming ME, Michael NL, Robb M, Cohen MS, Neuzil KM. Accelerating Development of SARS-CoV-2 Vaccines - The Role for Controlled Human Infection Models. N Engl J Med. 2020 Jul 1. doi: 10.1056/NEJMp2020076. Online ahead of print.

[42] Jarret C, Wilson R, O'Leary M, Eckersberger E, Larson H, SAGE working group on vaccine hesitancy. Strategies for addressing vaccine hesitancy - A systematic review. Vaccine. 2015; 33 (34): 4180-4190 AND World Health Organization. 2020. Key criteria for the ethical acceptability of COVID-19 human challenge studies. Available at https://apps.who.int/iris/bitstream/handle/10665/331976/WHO-2019-nCoV-Ethics_criteria2020.1-eng.pdf?ua=1; AND Dada S, McKay G, Mateus A, Lees S. Lessons learned from engaging communities for Ebola vaccine trials in Sierra Leone: reciprocity, relatability, relationships and respect (the four R's) BMC Public Health (2019) 19:1665 https://doi.org/10.1186/s12889-019-7978-4; AND Schoch-Spana M, Brunson E, Long R, Ravi S, Ruth A, Trotochaud M on behalf of the Working Group on Readying Populations for COVID-19 Vaccine. The Public's Role in COVID-19 Vaccination: Planning Recommendations Informed by Design Thinking and the Social, Behavioral, and Communication Sciences. 2020. The Johns Hopkins Center for Health Security. https://www.centerforhealthsecurity.org/ourwork/publications/the-publics-role-in-covid-19-vaccination. [43] Morefield H. The potential risk of chasing a covid-19 vaccine. Washington Post May 19, 2020. Available at https:/www.washingtonpost.com/outlook/2020/05/19/potential-risk-chasingcovid-19-vaccine/ 
Table: Selected ethically relevant dimensions affecting social and scientific value in accelerated vaccine development approaches

\begin{tabular}{|c|c|c|c|c|c|c|c|}
\hline \multirow[b]{2}{*}{ Approach } & \multicolumn{7}{|c|}{ Selected ethically relevant dimensions } \\
\hline & Speed & $\begin{array}{l}\text { Total number of } \\
\text { research } \\
\text { participants }\end{array}$ & $\begin{array}{l}\text { Risks to } \\
\text { participants and } \\
\text { vaccinees }\end{array}$ & Cost & $\begin{array}{l}\text { Feasibility- } \\
\text { research } \\
\text { capacity }\end{array}$ & $\begin{array}{l}\text { Feasibility- } \\
\text { pandemic } \\
\text { dynamics }\end{array}$ & $\begin{array}{l}\text { Social } \\
\text { Distrus } \\
\text { Negati } \\
\text { Percep }\end{array}$ \\
\hline $\begin{array}{l}\text { Consecutive } \\
\text { Phase I, II and III } \\
\text { Trials }\end{array}$ & $\begin{array}{l}\text { Years to trial } \\
\text { completion }\end{array}$ & $\begin{array}{l}\text { Thousands to } \\
\text { tens of } \\
\text { thousands }\end{array}$ & $\begin{array}{l}\text { Low and } \\
\text { carefully } \\
\text { monitored }\end{array}$ & High & $\begin{array}{l}\text { High: existing } \\
\text { experience } \\
\text { and } \\
\text { infrastructure }\end{array}$ & $\begin{array}{l}\text { Showing } \\
\text { efficacy } \\
\text { depends on } \\
\text { sufficient } \\
\text { incidence }\end{array}$ & $\begin{array}{l}\text { Usuall } \\
\text { familia } \\
\text { traject }\end{array}$ \\
\hline $\begin{array}{l}\text { Individually } \\
\text { randomized RCT } \\
\text { Combining } \\
\text { Phases II/III }\end{array}$ & & & & & & & \\
\hline $\begin{array}{l}\text { Cluster } \\
\text { randomized RCT } \\
\text { in Phase III }\end{array}$ & & & & & & & \\
\hline $\begin{array}{l}\text { EUA following } \\
\text { Phase I }\end{array}$ & & $\mathrm{N} / \mathrm{A}$ & & & $\mathrm{N} / \mathrm{A}$ & $\mathrm{N} / \mathrm{A}$ & \\
\hline $\begin{array}{l}\text { CHI study with or } \\
\text { without field trial }\end{array}$ & & & & & & & \\
\hline
\end{tabular}

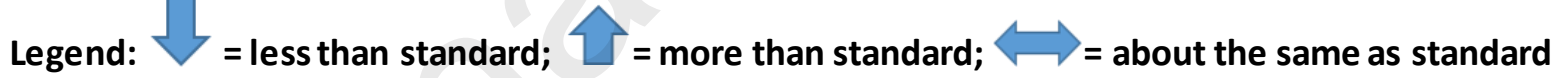
approach.

CHI- controlled human infection; EUA- Emergency Use Authorization; RCT - randomized-controlled trial. 


\section{Declaration of interests}

$\bigotimes$ The authors declare that they have no known competing financial interests or personal relationships that could have appeared to influence the work reported in this paper.

$\square$ The authors declare the following financial interests/personal relationships which may be considered as potential competing interests: 


\begin{abstract}
Background: A sense of urgency exists to develop vaccines against SARS Co-V-2, responsible for numerous global cases and deaths, as well as widespread social and economic disruption. Multiple approaches have been proposed to speed up vaccine development, including accelerated randomized controlled trials (RCT), controlled human challenge trials (CHI), and wide distribution through an emergency use authorization after collecting initial data. There is a need to examine how best to accelerate vaccine development in the setting of a pandemic, without compromising ethical and scientific norms.

Methods: Trade-offs in scientific and social value between generating reliable evidence about safety and efficacy while promoting rapid vaccine availability are examined along five ethically relevant dimensions: (1) confidence in and generalizability of data, (2) feasibility, (3) speed and cost, (4) participant risks, and 5) social risks.

Results: Accelerated individually randomized RCTs permit expeditious evaluation of vaccine candidates using established methods, expertise, and infrastructure. RCTs are more likely than other approaches to be feasible, increase speed and reduce cost, and generate reliable data about safety and efficacy without significantly increasing risks to participants or undermining societal trust.

Conclusion: Ethical analysis suggests that accelerated RCTs are the best approach to accelerating vaccine development in a pandemic, and more likely than other approaches to enhance social value without compromising ethics or science. RCTs can expeditiously collect rigorous data about vaccine safety and efficacy. Innovative and flexible designs and implementation strategies to respond to shifting incidence and test vaccine candidates in parallel or sequentially would add value, as will coordinated data sharing across vaccine trials. CHI studies may be an important complementary strategy when more is known. Widely disseminating a vaccine candidate without efficacy data will not serve the public health nor achieve the goal of identifying safe and effective SARS Co-V 2 vaccines.
\end{abstract}

